\title{
Identification of a cytokine network sustaining neutrophil and Th17 activation in untreated early rheumatoid arthritis
}

\author{
R Cascão ${ }^{1 \dagger}$, R A Moura $^{1 \dagger}$, I Perpétuo ${ }^{1}$, H Canhão ${ }^{1,2}$, E Vieira de Sousa ${ }^{1,2}$, A F Mourão ${ }^{1,3}$, A M Rodrigues ${ }^{1,2}$, \\ J Polido-Pereira ${ }^{1,2}$, M Viana Queiroz ${ }^{2}$, H S Rosário ${ }^{4}$, M M Souto-Carneiro $^{5}$, L Graca ${ }^{6^{*}}$, J E Fonseca ${ }^{1,2^{*}}$ \\ From 5th European Workshop on Immune-Mediated Inflammatory Diseases \\ Sitges-Barcelona, Spain. 1-3 December 2010
}

\section{Introduction}

Rheumatoid arthritis (RA) is a chronic inflammatory autoimmune disease characterized by sustained synovitis. Recently, several studies have proposed neutrophils and Th17 cells as key players in the onset and perpetuation of this disease.

\section{Aim}

To determine whether cytokines driving neutrophil and Th17 activation are dysregulated in very early rheumatoid arthritis patients with less than 6 weeks of disease duration and before treatment (VERA).

\section{Patients and methods}

Cytokines related to neutrophil and Th17 activation were quantified in the serum of VERA and established RA patients and compared with other very early arthritis (VEA) and healthy controls. Synovial fluid (SF) from RA and osteoarthritis (OA) patients was also analyzed.

\section{Results}

VERA patients had increased serum levels of cytokines promoting Th17 polarization (IL-1 $\beta$ and IL-6), as well as Th17-derived cytokines (IL-17A and IL-22), known to induce neutrophil-mediated inflammation, such as IL-8. In established RA this pattern is more evident within the SF. Early treatment with MTX or corticosteroids led to clinical improvement but with no impact on the cytokine pattern.

\section{Conclusions}

VERA patients already display increased levels of cytokines related with Th17 polarization and neutrophil recruitment and activation, a dysregulation also found in SF of established RA. Thus, our data suggest that a cytokine-milieu favoring Th17 and neutrophil activity is an early event in RA pathogenesis.

\section{Author details \\ 'Rheumatology Research Unit, Instituto de Medicina Molecular, Faculdade de Medicina da Universidade de Lisboa, Lisboa, Portugal. ${ }^{2}$ Rheumatology Dept., Centro Hospitalar de Lisboa Norte, EPE, Hospital de Santa Maria, Lisboa, Portugal. ${ }^{3}$ Rheumatology Dept., Centro Hospitalar de Lisboa Ocidental, EPE, Hospital Egas Moniz, Lisboa, Portugal. ${ }^{4}$ Microvascular Biology and Inflammation Unit, Instituto de Medicina Molecular, Faculdade de Medicina da Universidade de Lisboa, Lisboa, Portugal. ${ }^{5}$ Center for Neurosciences and Cell Biology, Autoimmunity Group, Coimbra, Portugal. ${ }^{6}$ Cellular Immunology Unit, Instituto de Medicina Molecular, Faculdade de Medicina da Universidade de Lisboa, Lisboa, Portugal.}

Published: 25 November 2010

\section{doi:10.1186/1479-5876-8-S1-P34}

Cite this article as: Cascão et al.: Identification of a cytokine network sustaining neutrophil and Th17 activation in untreated early rheumatoid arthritis. Journal of Translational Medicine 2010 8(Suppl 1):P34.

\footnotetext{
† Contributed equally

${ }^{1}$ Rheumatology Research Unit, Instituto de Medicina Molecular, Faculdade de Medicina da Universidade de Lisboa, Lisboa, Portugal

${ }^{6}$ Cellular Immunology Unit, Instituto de Medicina Molecular, Faculdade de

Medicina da Universidade de Lisboa, Lisboa, Portugal

Full list of author information is available at the end of the article
} 